in vivo $34: 1749-1758(2020)$

doi:10.21873/invivo.11968

\title{
A Feasibility Study for 3D-printed Poly(methyl methacrylate)-resin Tracheostomy Tube Using a Hamster Cheek Pouch Model
}

\author{
HARRY JUNG ${ }^{1}$, JI SEUNG LEE ${ }^{2}$, JUN HO LEE ${ }^{3}$, KI JOON PARK ${ }^{3}$, JAE JUN LEE ${ }^{4}$ and HAE SANG PARK ${ }^{1,3}$ \\ ${ }^{1}$ Institute of New Frontier Research Team, Hallym University, \\ Hallym Clinical and Translation Science Institute, Chuncheon, Republic of Korea; \\ ${ }^{2}$ Nano-Bio Regenerative Medical Institute, School of Medicine, Hallym University, Chuncheon, Republic of Korea; \\ ${ }^{3}$ Department of Otorhinolaryngology-Head and Neck Surgery, Chuncheon Sacred Heart Hospital, \\ College of Medicine, Hallym University, Chuncheon, Republic of Korea; \\ ${ }^{4}$ Department of Anesthesiology and Pain Medicine, College of Medicine, \\ Hallym University, Chuncheon, Republic of Korea
}

\begin{abstract}
Background/Aim: A three-dimensional (3D) printed tracheostomy tube has potential application for patients who require a specialized tube. The aim of this study was to evaluate the characteristics of various $3 D$ printing materials and determine their use in producing 3D-printed tracheostomy tube. Materials and Methods: Mechanical, chemical, and microbiological in vivo changes in the scaffolds were analyzed using a hamster cheek pouch (HCP) model. Results: The poly methyl methacylate (PMMA)-resin showed superior pre- and post-insertion mechanical properties and a relatively consistent lower biofilm formation compared with other scaffolds. PMMA-resin was successfully $3 D$-printed with dimensional accuracy without a support system. The use of a 3D-printed PMMA tracheostomy tube in a rabbit trachea showed no definite signs of infection, allergy or foreign body reaction. Conclusion: PMMA-resin can be proposed as an alternative for a 3D-printed tracheostomy tube material. In addition, we suggest HCPs as an in vivo model for evaluating indwelling medical devices.
\end{abstract}

Tracheostomy is a surgical procedure for securing the airway in cases of upper airway obstruction, prolonged assisted ventilation, and pulmonary toilet (1). In most cases,

This article is freely accessible online.

Correspondence to: Hae Sang Park, MD, Ph.D., Department of Otorhinolaryngology-Head and Neck Surgery, Chuncheon Sacred Heart Hospital, College of Medicine, Hallym University, 77, Sakjuro, Chuncheon, Gangwon-do, 24253, Republic of Korea. Tel: +82 332405181, Fax: +82 332412909, e-mail: hs-piao@hanmail.net

Key Words: 3D printing, resin, tracheostomy, tube, hamster. commercially available and prefabricated tracheostomy tubes (TTs) are used to keep the tracheostoma open (2). However, they often do not fit in patients, such as obese or pediatric patients, patients with abnormal anatomy of the neck and thorax (e.g., Duchenne muscular dystrophy), or those with laryngotracheal disease (e.g., tracheal stenosis, or cancer) (2-7). Although commercially available nonstandard (extended-length) TTs do exist, it is often impractical for hospitals to carry all possible types of TTs because of the cost, expiration dates, and availability (3). Therefore, in most cases, nonstandard TTs cannot be used immediately when they are needed.

With the great progress made in $3 \mathrm{D}$ printing and computer-aided design (CAD), these technologies have been widely applied in the medical field, from simulation and preoperative planning to live-cell printing (8). Personalized implants or casts produced by $3 \mathrm{D}$ printing have been successfully introduced in the orthopedic, craniofacial reconstruction, and dental fields (9-14). However, there have only been a few reports on the use of 3D printing to design airway prostheses, such as TTs $(2,15,16)$. Although a $3 \mathrm{D}$ model of the patient's upper airway was used to design a personalized tube in these studies, a real tracheostomy tube was fabricated using conventional manufacturing methods based on this 3D model. Therefore, to the best of our knowledge, there have been no personalized TTs printed by a 3D printer yet. Because 3D printing can be more convenient, more flexible, and faster than conventional manufacturing techniques (17), a 3D-printed personalized TT has potential application in patients who need a specialized tube because of medical reasons.

The purpose of this study was to evaluate the characteristics of various $3 \mathrm{D}$ printing materials using a hamster cheek pouch (HCP) model and determine their use as a 3D-printed TT. We used polycaprolactone (PCL), polylactic acid (PLA) and poly 
methyl methacylate (PMMA) resins for applying two different types of 3D printing methods, which are widely used in biomedical applications: fused deposition modeling (FDM), and digital light processing (DLP) (18). PCL and PLA are biodegradable synthetic materials for FDM that have superior mechanical strengths, and comparable biocompatibilities (19, 20). PMMA-resin is a photo-curable, 3D-printable resin for DLP, which offers many advantages in dental prosthesis manufacturing, such as 3D-printed artificial teeth, denture bases, provisional crowns, and dental bridge material (13, 14, 21-24). As TT is an indwelling catheter placed in the tracheal lumen, chemical and mechanical changes of the tube as well as biofilm formation are important factors for determining safety for clinical application. To evaluate these properties, we introduced an HCP model. Additionally, animal tracheostomy was conducted to evaluate the possibility of a 3D-printed TT.

\section{Materials and Methods}

Selecting tube material: Use of HCP model

$C A D$ and $3 D$ printing for specimen preparation. A cylindrical scaffold was generated using CAD software (SOLIDWORKS, Dassault Systèmes SolidWorks Corp., Waltham, MA, USA) (outer diameter of $4.1 \mathrm{~mm}$, inner diameter of $3.1 \mathrm{~mm}$, and height of 10 $\mathrm{mm}$ ). The PLA (CAS number 26100-51-6, Sigma-Aldrich, St. Louis, MO, USA) and PCL (Purasorb ${ }^{\circledR}$ PC 12, Corbion Purac, Amsterdam, the Netherlands) were placed in a heating jacket and extruded through a steel nozzle using an FDM 3DP-Printer (T\&R Biofab, Siheung-si, Gyeonggi-do, Republic of Korea) (temperature PLA, $190^{\circ} \mathrm{C}$; PCL $110^{\circ} \mathrm{C}$ ). The inner diameter of the nozzle was $500 \mu \mathrm{m}$, and the thickness of each layer was $400 \mu \mathrm{m}$. The PMMAresin (NextDent ${ }^{\mathrm{TM}} \mathrm{C} \& \mathrm{~B}$, NextDent, Soesterberg, the Netherlands) specimens were manufactured using a DLP 3D printer (MiiCraft Ultra 50X, MIICRAFT, Jena, Germany). The following printing parameters were used: a printing thickness of $15 \mu \mathrm{m}$ and curing time of $1.5 \mathrm{~s}$. After printing, the printed scaffold was briefly rinsed with deionized water to remove any unreacted solution. A commercially available polyvinyl chloride (PVC) TT (Portex ${ }^{\circledR}$ Blue Line ${ }^{\circledR}$ Uncuffed Tracheostomy Tubes, Smiths Medical, Inc., Minneapolis, MN, USA) was used for the control group. It was cut to achieve an outer diameter of $9.7 \mathrm{~mm}$, inner diameter of $7 \mathrm{~mm}$, and height of $10 \mathrm{~mm}$.

SEM. A low-vacuum scanning electron microscope (S-350N, Hitachi, Tokyo, Japan) located at the Korea Basic Science Institute, Chuncheon, Republic of Korea, was used. The scaffold samples were coated with a thin $10 \mathrm{~nm}$ layer of gold/palladium using an ion sputterer (1010, Hitachi, Tokyo, Japan) at a discharge current of 15 $\mathrm{mA}$ for $30 \mathrm{~s}$. Micrographs were obtained at an accelerating voltage of $3 \mathrm{kV}$.

Cell viability analysis using the CCK-8 assay. NIH3T3 fibroblasts were cultured in DMEM/High Glucose (Welgene Inc., Gyeongsan, Korea) with $10 \%$ fetal bovine serum (FBS; Gibco, Grand Island, NY, USA) and incubated at $37^{\circ} \mathrm{C}$ in an atmosphere of $5 \% \mathrm{CO}_{2}$. The proliferation activity of NIH3T3 fibroblasts was quantified at day 2 using a cell viability assay kit (Quanti-Max, Dojindo Molecular Technologies, Inc., Kumamoto, Japan). The cells were rinsed in

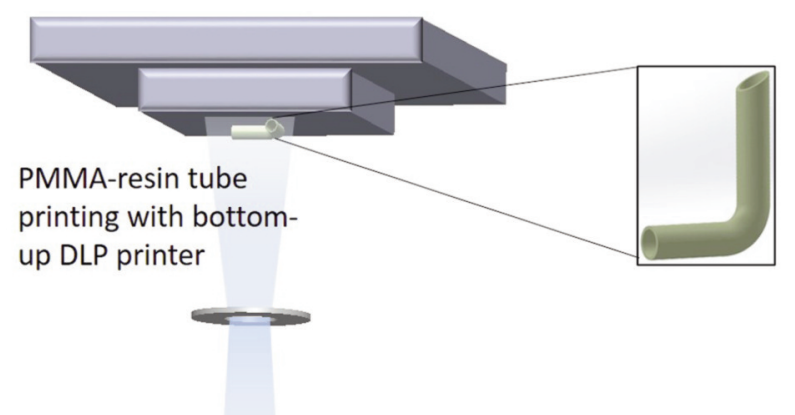

UV Lighting

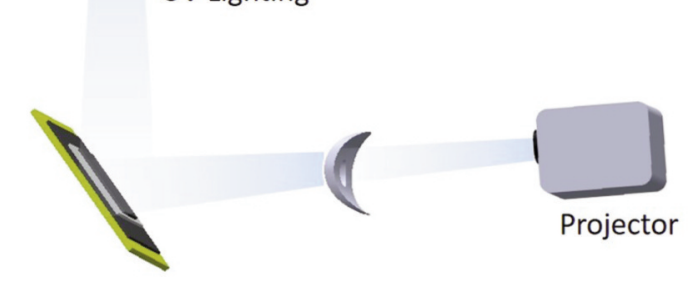

Figure 1. Scheme of the $3 D$ printing process for 3D-printed PMMAresin tube. The PMMA-resin tracheostomy tube was manufactured in a layer-by-layer through photopolymerization of the PMMA-resin by ultraviolet light. PMMA: Poly methyl methacylate; DLP: digital light processing; UV: ultraviolet.

phosphate-buffered saline (PBS, pH 7.4) and then submersed in $10 \mu \mathrm{l}$ CCK-8 reagent diluted with $100 \mu \mathrm{l}$ of medium at $37^{\circ} \mathrm{C}$ for $30 \mathrm{~min}$. The absorbance readings at $450 \mathrm{~nm}$ were obtained using a GloMax system (Promega, GloMax Navigator Microplate Luminmeter ${ }^{\circledR}$, Madison, WI, USA) at 72 and $96 \mathrm{~h}$.

Insertion of the scaffold into an HCP. Twelve male Golden Syrian hamsters (Japan SLC, Nagoya, Japan) with approximate weight of $120 \mathrm{~g}$ were used. The animals were anesthetized using an intraperitoneal injection of a mixture of $1 \mathrm{ml}$ ketamine $(8.3 \mu \mathrm{l} / \mathrm{g}$, Huons, Seongnam, Gyeonggi-do, Republic of Korea) and $0.5 \mathrm{ml}$ Rumpun $^{\circledR}(4.1 \mu \mathrm{l} / \mathrm{g}$, xylazine chloride, Bayer Korea, Seoul, Republic of Korea). After exposing the cheek pouches, a single scaffold was inserted into each cheek pouch. Each group was composed of three animals, and a total of six scaffolds were used for each group. The cheek pouch opening was closed by purse-string suturing with 3-0 silk (Ethicon Inc., Somerville, NJ, USA). After 2 weeks of insertion, the scaffolds were removed from the HCPs. This study was approved by the institutional review board of Hallym University (Hallym 2018-67), Chuncheon, Republic of Korea.

Fourier transform infrared (FTIR) spectroscopy. A Frontier Optica (PerkinElmer Inc., Buckinghamshire, UK) spectrometer was used to analyze the attenuated total reflection (ATR)-FTIR spectra of the scaffolds to confirm the secondary chemical transition of the scaffolds after the in vivo insertion. The spectra were recorded from 8,300 to $350 \mathrm{~cm}^{-1}$ with a $0.4 \mathrm{~cm}^{-1}$ resolution and 32 scans.

Mechanical tests. The uniaxial compressive property of each scaffold was measured using a universal testing machine (QM100S, qmesys, Kyounggi, Republic of Korea) with a $200 \mathrm{kN}$ capacity load cell and crosshead loading rate of $0.5 \mathrm{~mm} \mathrm{~min}^{-1}$. The force required 
to pull the specimen apart and the magnitude of the sample strength before breaking were measured. Three samples of each scaffold were used for this analysis.

Biofilms assay. Each scaffold was removed from the HCP 2 weeks after the insertion, and the cheek pouch mucosa tissues were harvested. The cheek pouch mucosa harvested the outer and the luminal surfaces of the scaffolds/were rinsed with $10 \mathrm{ml}$ of sterile $0.9 \%$ saline solution. The microbial concentration of the rinsing solution was determined using the standard microbiological serial dilution method and plating on different culture agar media. The growth medium for Pseudomonas aeruginosa ( $P$. aeruginosa) was made by suspending $38 \mathrm{~g}$ of the Pseudomonas agar $\mathrm{F}$ (BD Biosciences, Bedford, MA, USA) in 11 of base buffer containing $10 \mathrm{~g}$ glycerol. The growth medium for Staphylococcus aureus ( $S$. aureus) was made by suspending $63 \mathrm{~g}$ of the Staphylococcus medium 110 (BD Biosciences) in $950 \mathrm{ml}$ of deionized water. After boiling for $1 \mathrm{~min}$, the powder was completely dissolved and it was autoclaved at $121^{\circ} \mathrm{C}$ for $15 \mathrm{~min}$. The rinsing solutions for the scaffolds and oral mucosa tissues were inoculated into the growth media for $24 \mathrm{~h}$ at $37^{\circ} \mathrm{C}$ and the colony-forming units (CFUs) were counted manually. Candida albicans (C. albicans) was analyzed using 3M Petrifilm (3M Petrifilm, St. Paul, MN, USA) and incubated at $37^{\circ} \mathrm{C}$ for $48 \mathrm{~h}$.

Feasibility test for 3D printed tracheostomy tube

$3 D$ printing. Computed tomography (CT) images of a 20 -week-old rabbit were taken to observe the airway anatomy for the $3 \mathrm{D}$ design of the TT. It was confirmed that the inner diameter was approximately $6.5 \mathrm{~mm}$, and the outer diameter was approximately $7.5 \mathrm{~mm}$. Based on this result, a 3D model of TT for the rabbit was generated (SOLIDWORKS, Dassault Systèmes SolidWorks Corp., Waltham, MA, USA). The 3D model of the TT was composed of a tube shaft and neck flange, designed as an angled tube $\left(90^{\circ}\right)$ with curved and straight portions. The tube shaft was manufactured with a DLP 3D printer (MiiCraft Ultra 50X, MIICRAFT, Jena, Germany) using the PMMA-resin, and printing parameters were the same as for a cylindrical scaffold (Figure 1). The neck flange was manufactured with silicone (Dow Corning ${ }^{\circledR}$ SE 1700, Midland, MI, USA) using an FDM 3D-Discovery ${ }^{\mathrm{TM}}$ 3D bio-printer (regenHu, Fribourg, Switzerland). The inner diameter of the nozzle was 0.25 $\mathrm{mm}$, and the thickness of each layer was $0.22 \mathrm{~mm}$.

Tracheostomy. New Zealand rabbits (20 weeks old and weighing $3.5 \sim 3.7 \mathrm{~kg}, \mathrm{n}=2$ ) were used. This study was approved by the Institutional Review Board of Hallym University, Chuncheon, Republic of Korea (Hallym 2017-67). The animals were anesthetized by an intramuscular injection of a mixture of $2 \mathrm{ml}$ Ketamine $\left(0.6 \mathrm{ml} / \mathrm{kg}\right.$, Huons) and $1 \mathrm{ml}$ Rumpun ${ }^{\circledR}(0.3 \mathrm{ml} / \mathrm{kg}$ xylazine chloride, Bayer, Republic of Korea). After exposing the trachea, one cartilage ring was removed, and the prepared $3 \mathrm{D}$ printed TT was inserted into the tracheostoma. Neck straps were used to keep the tracheostomy tube in place. Bronchoscopy (rigid 4 $\mathrm{mm} 0^{\circ}$ endoscope, Karl Storz, Tuttlingen, Germany) and CT scan were performed.

Histology. The trachea specimen was retrieved 3 weeks after the tracheostomy. The specimen was embedded in a paraffin block and sectioned into $5 \mu \mathrm{m}$ thick slices, placed on slides, and then stained with hematoxylin-eosin (H\&E).
Statistical analysis. All data are presented as the mean \pm standard deviation. A statistical analysis of the experimental results was performed using the Statistical Package for Social Sciences software program (SPSS) (SPSS Inc., Chicago, IL, USA). A $p$-value was generated with a Student's $t$-test, with the statistical significance set at $* p<0.05, * * p<0.01$, and $* * * p<0.005$.

\section{Results}

SEM data and CCK-8 assay. Figure 2 shows the external appearance and SEM images of the scaffolds. Because the FDM printer builds structures layer by layer from the bottom up, we could observe the deposited PLA or PCL fibers in the scaffolds using SEM images. However, the PMMA-resin scaffold printed by the DLP printer showed no fiber structures. Except for this difference in printing methods (FDM versus DLP), no definite contaminating foreign materials or cracks on the surface were found in any of the different types of scaffolds. The cell viability values for the NIH3T fibroblasts were higher in all groups compared to the control group, indicating that no toxic effects could be attributed to the scaffolds. There were no statistically significant cell viability differences based on time between the scaffolds (Figure 2).

In vivo study using $\mathrm{HCPs}$

FTIR spectroscopy. The FTIR spectra of each scaffold before insertion and two weeks after insertion showed no significant difference in the functional groups. These results indicated that there was no structural transition in the scaffold during the in vivo insertion (Figure 3 ).

Mechanical properties. The compressive strengths before implantation were $679.8 \pm 28.6 \mathrm{kPa}$ in the PVC scaffolds, $4977.1 \pm 4.2 \mathrm{kPa}$ in the PCL scaffolds, $7121.5 \pm 6.4 \mathrm{kPa}$ in the PLA scaffolds, and $9734.9 \pm 4.2 \mathrm{kPa}$ in the PMMA-resin scaffolds. After two weeks of implantation in the HCP, each scaffold showed decreased compressive strength compared to the pre-insertion value. These were $555.0 \pm 7.1 \mathrm{kPa}$ in the PVC scaffolds, $3386.4 \pm 5.7 \mathrm{kPa}$ in the PCL scaffolds, $6678.8 \pm 16.3 \mathrm{kPa}$ in the PLA scaffolds, and $7428.5 \pm 2.8 \mathrm{kPa}$ in the PMMA-resin scaffolds. The 3D printed PCL, PLA, and PMMA-resin scaffolds demonstrated higher pre- and post-insertion compressive strengths than the conventional PVC scaffold. In addition, the PMMA-based resin showed the highest pre- and post-insertion compressive strengths (Figure 3).

Biofilm assay. Biofilms remain a common problem associated with indwelling medical devices, such as TTs. Therefore, in this study, $S$. aureus (gram-positive bacteria) and $P$. aeruginosa (gram-negative bacteria) were selected as experimental strains to evaluate the antibacterial activities of the various scaffolds. Biofilms appeared to form on both the surface and inner lumen of the scaffold in 
A

Outer

Inner

Upper

PVC
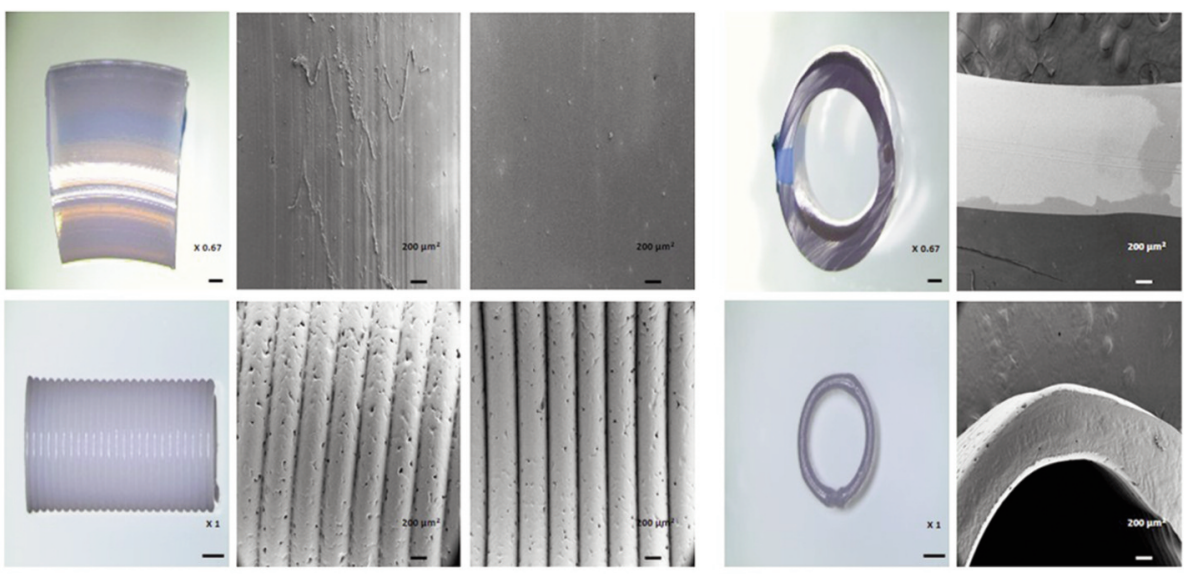

PCL

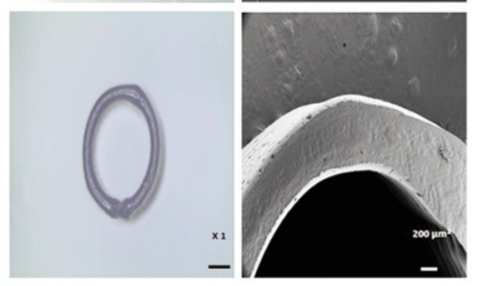

PLA
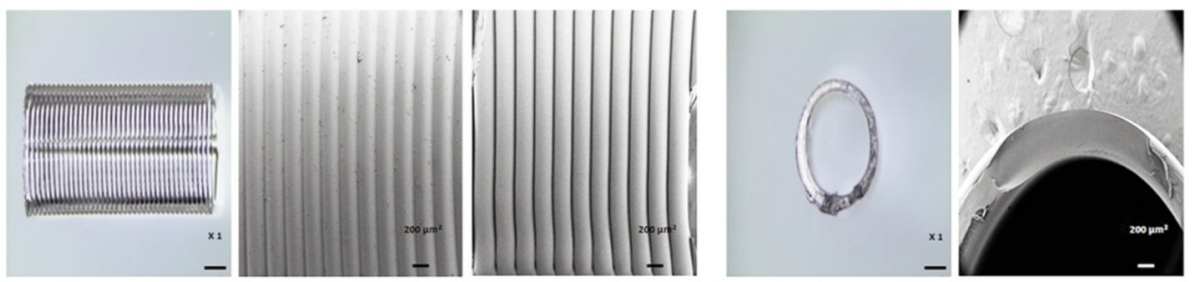

PMMA-

Resin
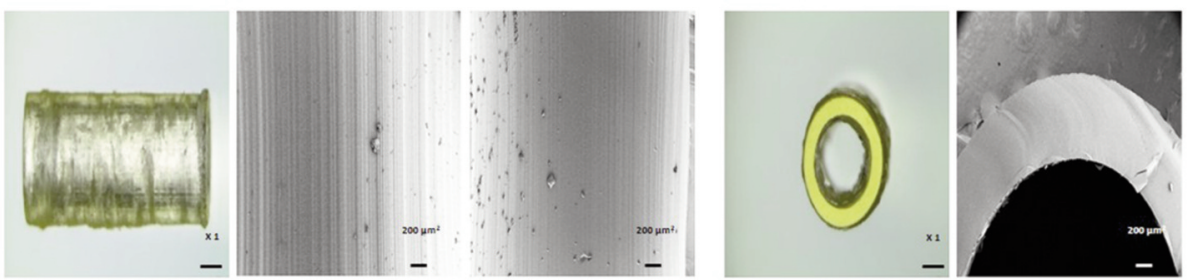

B

口Control QPVC QPCL QPLA QPMMA-Resin

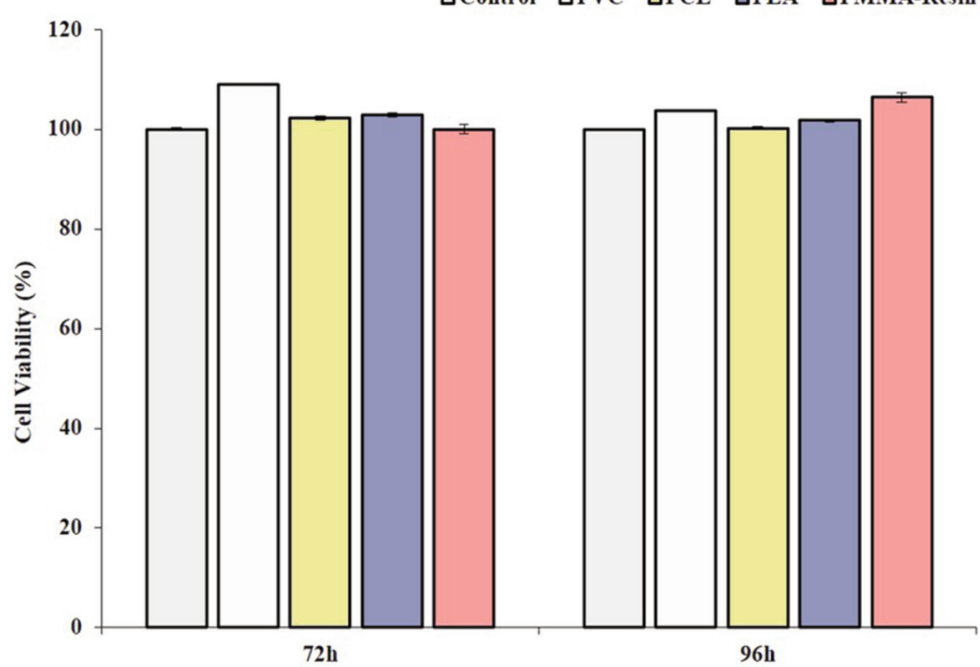

Figure 2. Images of the scaffolds and cell viability differences between them. A: External appearance and SEM images of the scaffolds. The PCL and PLA scaffolds printed by the FDM printer showed deposited fiber structures. The PMMA-resin scaffold printed by the DLP printer showed no fiber structures. B: Cell viability analysis using CCK-8 assay. Each scaffold showed no cytotoxicity. PVC: Polyvinyl chloride; PCL: polycaprolactone; PLA: polylactic acid; PMMA: poly methyl methacylate; FDM: fused deposition modeling; DLP: digital light processing. $\times 1$ (Light microscope), $\times 10,000 \mu^{2}($ SEM). 
A

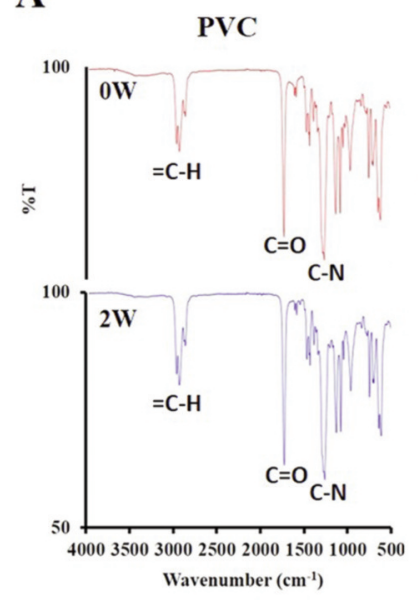

PLA
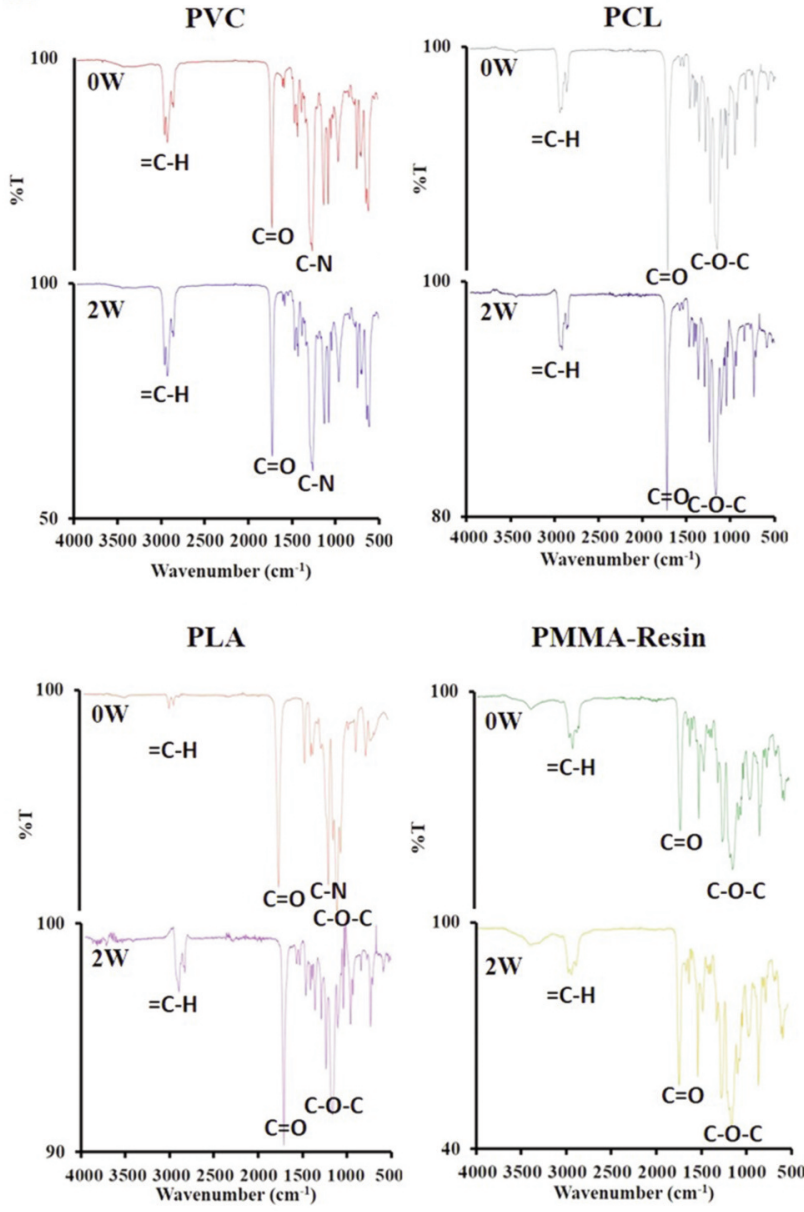

PMMA-Resin

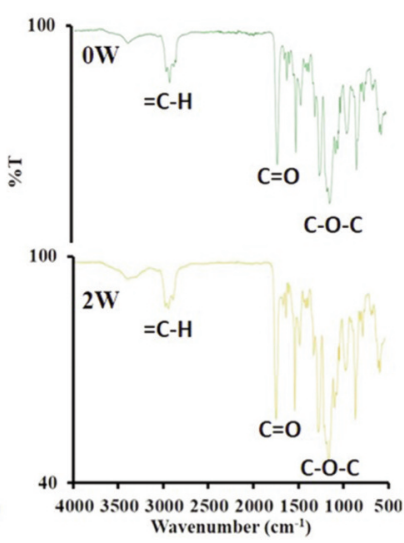

B

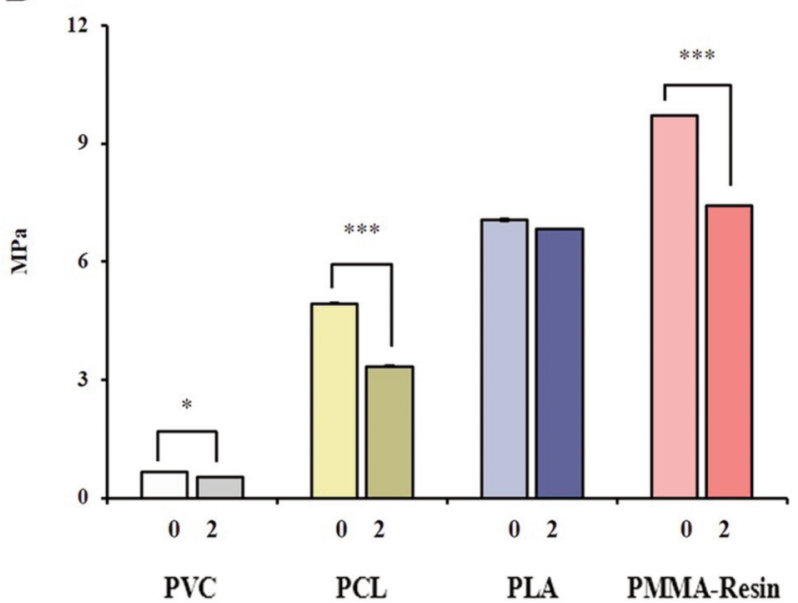

Figure 3. In vivo study using hamster cheek pouches. A: FTIR analysis. Each scaffold showed no definite chemical changes during in vivo insertion B: Mechanical properties. The PMMA-resin showed superior pre- and post-insertion mechanical properties. PVC: Polyvinyl chloride; PCL: polycaprolactone; PLA: polylactic acid; PMMA: poly methyl methacylate. $* p<0.05$, and $* * * p<0.005$. the SEM images. A significant increase in the number of adherent bacterial cells was obvious in the PVC scaffold. We could observe cocci-, rod-shaped bacteria, and Candida spp. embedded in the biofilm. The PMMA-resin demonstrated an obviously decreased number of bacterial cells compared to the other scaffolds (Figure 4). The CFU counts for $S$. aureus and $P$. aeruginosa were significantly higher in the PVC scaffold than in the PCL, PLA, and PMMA-resin scaffolds (Figure 4). The PMMA-resin scaffold showed the lowest $S$. aureus count among the four types of scaffolds $(p<0.05)$, and the $P$. aeruginosa count for the PLA scaffold was significantly lower than those of the other scaffolds $(p<0.05)$. However, the PCL scaffold showed the highest bacterial counts among the $3 \mathrm{D}$ printed scaffolds (PCL, PLA, and PMMA-resin). The CFU counts for $S$. aureus and $P$. aeruginosa in the HCP tissues were similar to those of the scaffolds. The CFU count for $C$. albicans was significantly higher in the PLA scaffold than in the other scaffolds (PVC, PCL, and PMMA-resin scaffold), and the PMMA-resin scaffold showed the lowest value $(p<0.005)$. However, $C$. albicans was not detected in the cheek pouch tissues (Figure 4).

Feasibility test for $3 D$ printed TT: Printabilty \& clinical usefulness. Based on the results of the in vivo insertion tests, PMMA-resin was finally selected as the material for the 3D printed tracheostomy tube for the following reasons. 1) The PMMA-resin showed pre- and post-insertion mechanical properties that were superior to those of the other scaffolds. 2) It also showed no definite cytotoxic effect. 3) There were no definite chemical changes during the in vivo insertion, and 4) it showed a relatively consistently lower biofilm formation than the other scaffolds. Figure 5 shows the gross appearance of the 3D-printed tube. TT, an angled cylinder, was successfully 3D-printed without support (sacrificial mold). The mean length, inner/outer diameter, and the angle of the 3D-printed TT are presented in Table I. Three samples were tested. All parameters were significantly close to the CAD design. During tracheostomy, the 3D-printed PMMAresin tube was inserted into the rabbit trachea without any difficulty (Figure 5). The bronchoscopy indicated that the PMMA-resin tube was inserted in the proper position in the tracheal lumen, and the diameter of the tube was suitably adapted to the diameter of the trachea. Mild granulation tissue was noticed around the stoma three weeks after the tracheostomy. However, the cannula was still properly placed in the tracheal lumen (Figure 6). The axial, sagittal, and 3Dreconstructed $\mathrm{CT}$ scan images also showed a properly positioned TT with no signs of associated complications, such as infection, tube displacement, or tracheal stenosis. The histology of the tracheal specimen after three weeks demonstrated the thickening of the epithelium and inflammatory tissue debris around the anterior portion of the 
A PVC

PCL

PLA

PMMA-Resin
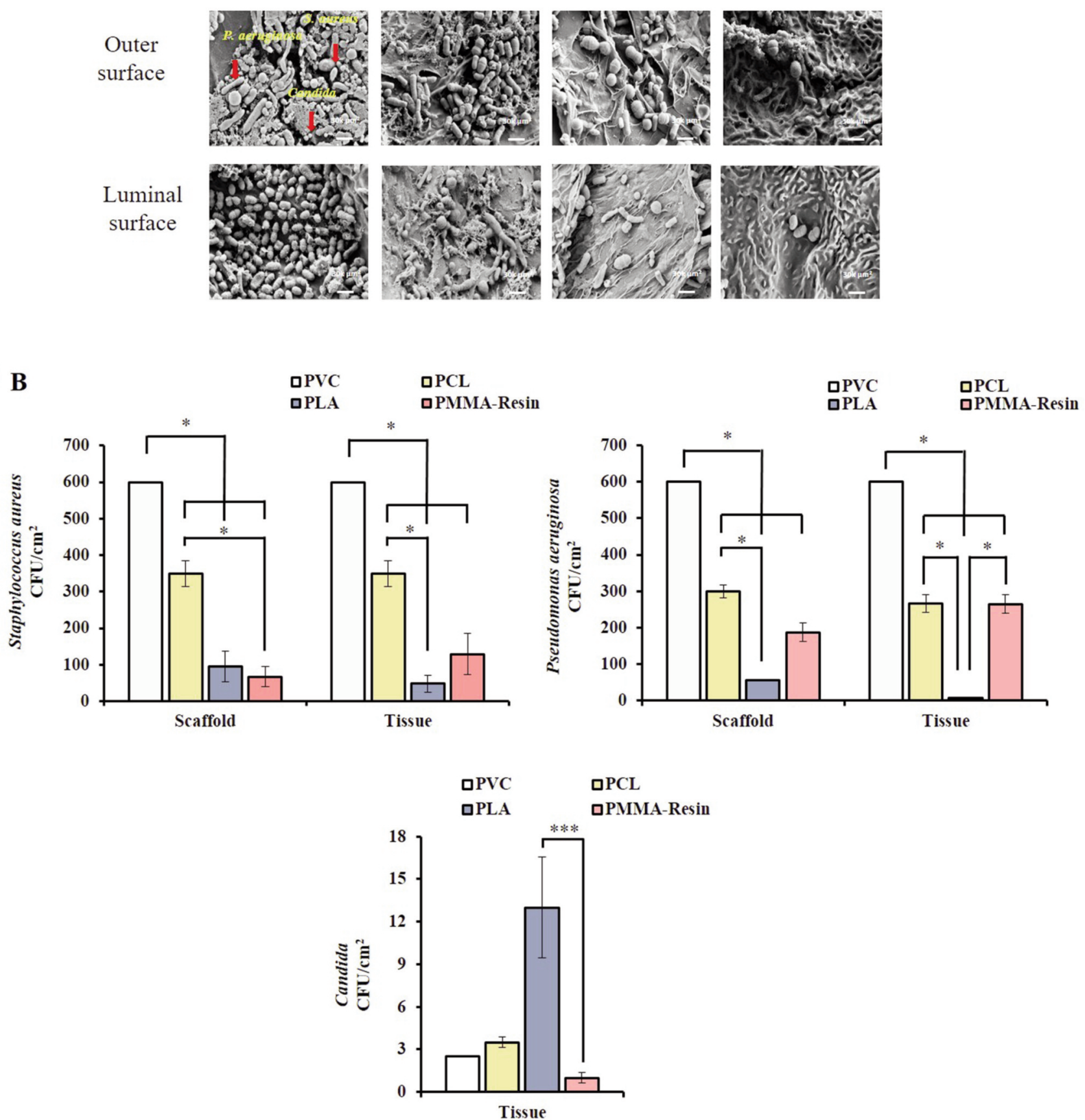

Figure 4. Biofilms assay on inserted scaffolds \& cheek pouch mucosa. A: SEM images of the outer and luminal surface of the scaffolds. B: CFU counts for S. aureus, P. aeruginosa, and C. albicans. CFU: Colony-forming unit. $\times 30,000 \mu \mathrm{m}^{2}$ (SEM). *p<0.05, and *** $p<0.005$.

trachea (stoma site). However, the normal structure (mucosa, submucosa, and cartilage) of the tracheal lumen was relatively well maintained. The posterior-lateral tracheal wall showed no definite inflammatory cells with normal luminal structure (Figure 6).

\section{Discussion}

This was the first study of a 3D-printed TT to combine the use of a computer-aided 3D model and biomaterial 3D printing. There are several requirements for ideal TTs, such 
A
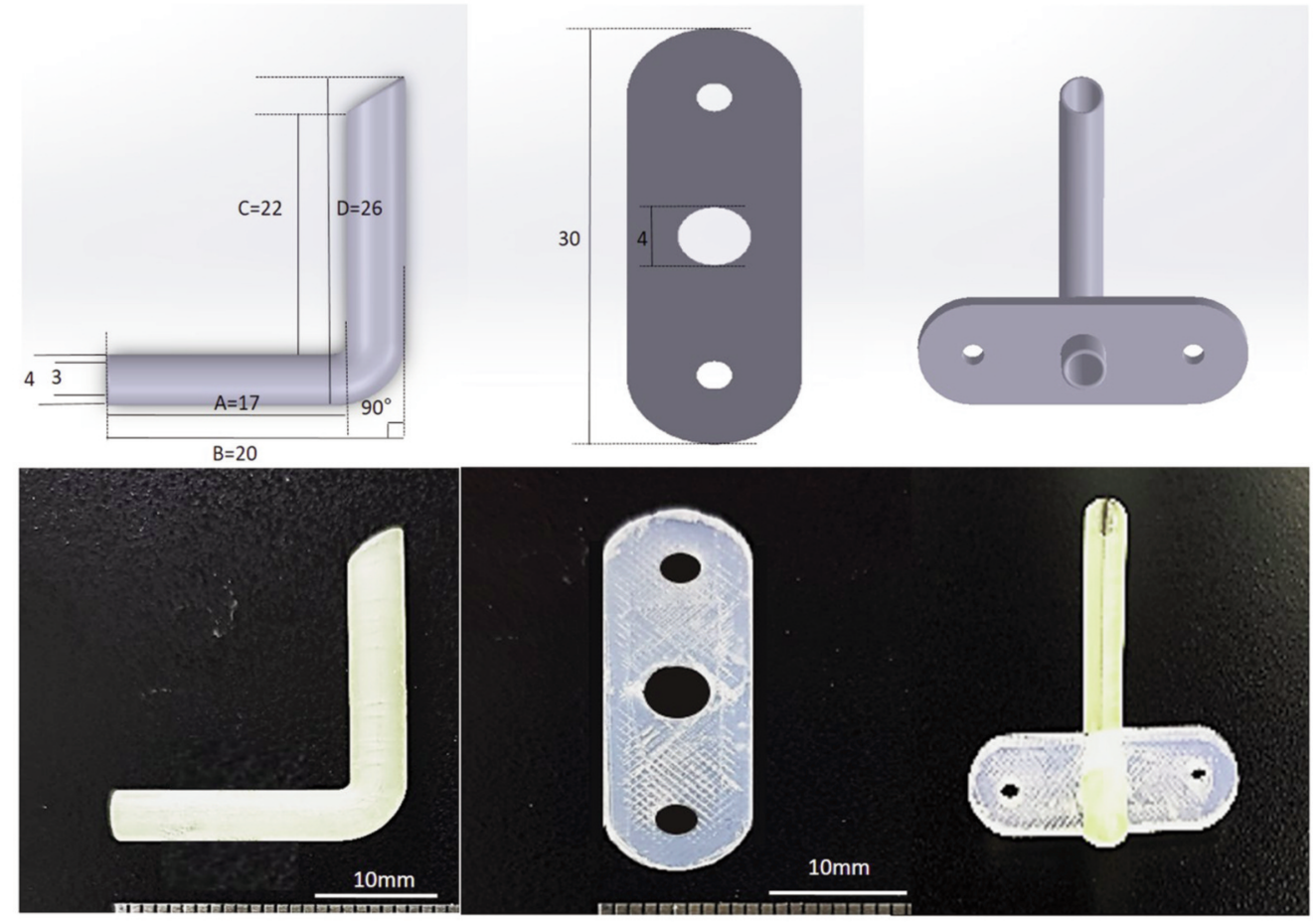

B

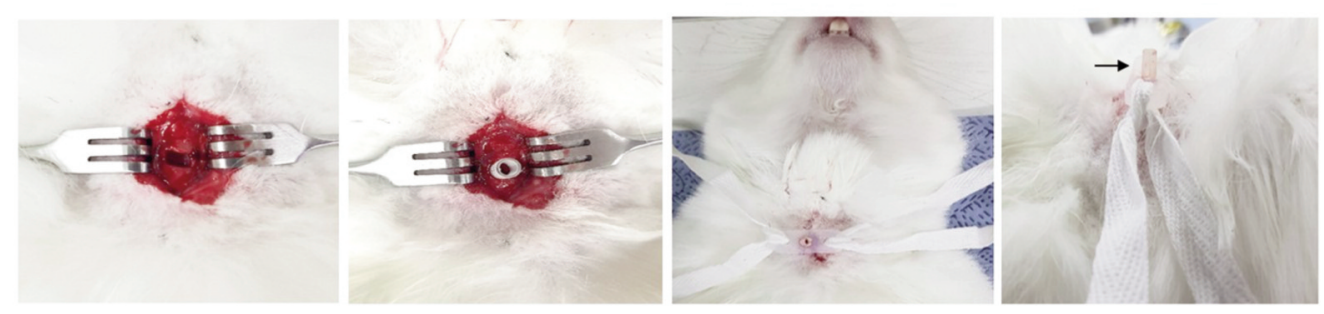

Figure 5. Tracheostomy with 3D printed tracheostomy tube. (A) CAD model and gross appearance of the 3D printed PMMA resin tube, (B) tracheostomy procedure in New Zealand rabbit. Arrow indicates inserted tube. PMMA: Poly methyl methacylate.

as proper rigidity for maintaining a tracheal lumen, lower cytotoxic effects on the tracheal mucosa, and anti-biofilm activity for preventing associated complications, such as wound infections, respiratory infections, tube obstruction, and tracheal stenosis (25). In addition, any chemical and mechanical changes in the tube during insertion are important issues, as TT is an indwelling medical prosthesis that lasts at least one week and up to several months in the tracheal lumen.

To evaluate these aspects, we introduced an HCP model in this study. It is best to evaluate changes in the TT under the same physiological conditions (tracheostomized animal), however, this method has several disadvantages, including costliness, and invasiveness of the surgery. HCPs are bilateral invaginations of the oral mucosa, and one of the most wellcharacterized animal models for oral carcinogenesis, and oral mucositis $(26,27)$. Since a major role of the HCP is storing food and bedding, we used HCPs as a storage/insertion space
Table I. Characteristics of the $3 D$ printed PMMA tube.

\begin{tabular}{lcc}
\hline$(\mathrm{mm})$ & 3D Model & PMMA tube \\
\hline Length A & 17 & $17.10 \pm 0.05$ \\
Length B & 20 & $20.13 \pm 0.08$ \\
Length C & 22 & $22.07 \pm 0.03$ \\
Length D & 26 & $26.07 \pm 0.06$ \\
Inner diameter & 3 & $3.03 \pm 0.03$ \\
Outer diameter & 4 & $4.03 \pm 0.03$ \\
\hline
\end{tabular}

PMMA: Poly methyl methacylate.

for scaffolds. We could easily carry scaffolds in the HCPs for two weeks by simple purse-string suturing of the HCP opening. HCPs could provide in vivo environments similar to the tracheal lumen, including secretion (saliva) from the oral mucosa, and normal bacterial flora. From this point of view, 
A

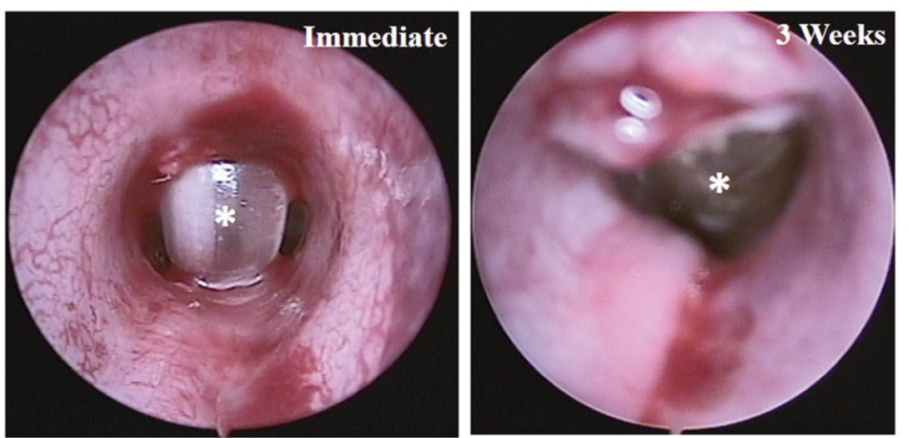

B
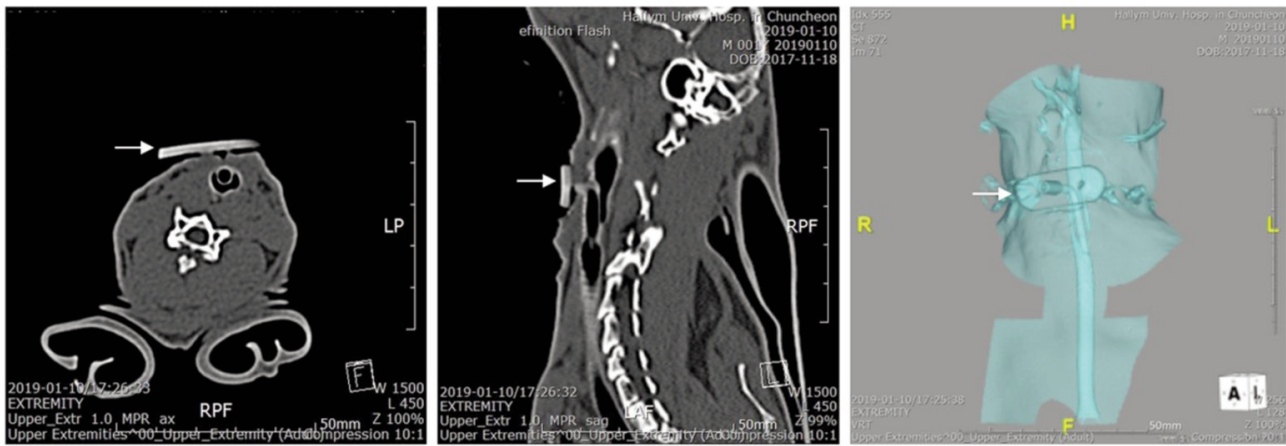

C
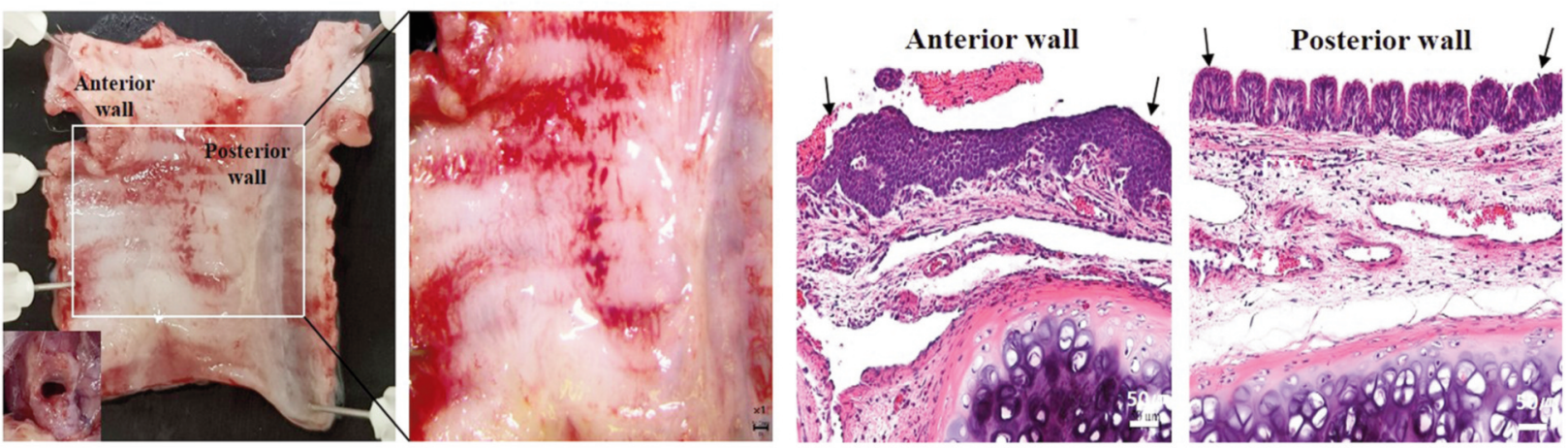

Figure 6. Assessments of post-tracheostomy procedure. A: Immediate bronchoscope findings and 3 weeks after tracheostomy. The PMMA-resin tube was inserted in the proper position in the tracheal lumen. Mild granulation tissue was noticed around the stoma three weeks after the tracheostomy. Asterisk indicating the tube. B: CT images of the rabbit 3 weeks after tracheostomy. Arrow indicating the tube, C: Gross findings and H\&E-stained sections of harvested trachea 3 weeks after tracheostomy. Arrows indicate tracheal epithelium. Thickening of the epithelium was observed on the tracheal anterior wall (stoma site). $\times 1$ (gross images), ×200 (H\&E). PMMA: Poly methyl methacylate; H\&E: hematoxylin and eosin.

we could suggest HCPs as an in vivo model for evaluating indwelling medical devices, such as a tracheostomy tube, and an oral/dental prosthesis. Using HCP models, we observed that none of the materials showed significant chemical changes during insertion. In addition, the PMMA-resin showed the highest compressive strength.

Antibacterial and antifungal properties are another concern when selecting 3D-printable materials for a TT. S. aureus, Staphylococcus epidermidis, P. aeruginosa, and Candida spp. have been identified as the most frequent pathogens colonizing the inner lumen of TTs (28). Biofilms are associated with upper respiratory infections, TT obstruction, and wound infections by Staphylococcus and Pseudomonas spp. $(25,29)$. Therefore, there have been several new trials to decrease wound infections by decreasing the presence of biofilms (17). From this point of view, we evaluated the biofilm formation in each of the scaffolds. The PLA and PMMA-resin scaffolds demonstrated lower bacterial counts for $S$. aureus and $P$. aeruginosa compared to the other scaffolds. However, PLA showed the highest Candida count. Taken together, the PMMA-resin showed relatively consistently lower CFU counts for $S$. aureus, $P$. aeruginosa, and $C$. albicans. Regarding the characteristics of the material, it has been known that the hydrophobicity, 
roughness, surface chemistry, and surface free energy of an implant play important roles in biofilm formation $(30,31)$. In this study, PCL and PLA showed the highest CFU counts for $S$. aureus, $P$. aeruginosa, and $C$. albicans. These results could be attributed to the surface roughness differences between the scaffolds according to the 3D-printing method (FDM, DLP), as well as the difference in the physicalchemical properties of the material (31).

Based on these results, PMMA-resin was finally selected as the material for a $3 \mathrm{D}$ printed TT. The 3D-designed model was successfully 3D-printed without a support system using a DLP printer. In the rabbit tracheostomy experiment, the 3D-printed PMMA-resin tube was easily inserted into the rabbit trachea. In addition, it was confirmed that the tube was properly positioned in the tracheal lumen for three weeks without complications. The histology also revealed no definite signs of infection, allergy or foreign body reactions in the tracheal mucosa to the PMMA-resin.

PMMA-resin can be suggested as an alternative 3Dprinted TT material that can be used in a DLP 3D printer: 1) The PMMA-resin showed superior pre- and post-insertion mechanical properties. 2) It showed no definite cytotoxicity. 3) There were no definite chemical changes and lower biofilm formation during the in vivo insertion, and 4) It showed dimensional accuracy in the 3D-printing of TTs. Although this study showed the considerable potential of a PMMA-resin TT, there are some limitations to clinical applications. Because this investigation was designed as a preliminary study, it might be relatively insufficient in a long-term evaluation. Therefore, before clinical application, long-term and larger animal studies are warranted.

\section{Conflicts of Interest}

The Authors have no conflicts of interest to declare regarding this study.

\section{Authors' Contributions}

Harry Jung: Methodology, Formal analysis, Investigation, Visualization, Writing original draft; Ji Seung Lee: Methodology, Investigation, Visualization, Validation; Jun Ho Lee: Investigation, resources; Ki Joon Park: Formal analysis, resources; Jae Jun Lee: Project administration, Data curation; Hae Sang Park: Conceptualization, Data curation, Supervision, Funding acquisition, Writing review \& editing.

\section{Acknowledgements}

This research was supported by the National Research Foundation of Korea (NRF) grant funded by the Ministry of Science, Information and Communication Technologies and Future Planning of the Korea government (NRF-2017M3A9E8033206); Hallym University Research Fund 2018 (HURF-2018-21).

\section{References}

1 Miller FR, Guay ME, Bauer T and Tucker HM: Long-term flap tracheostomy in a pediatric animal model. Arch Otolaryngol Head Neck Surg 121: 743-748, 2015. PMID: 7598850. DOI: 10.1001/archotol.1995.01890070029007

2 de Kleijn BJ, Kraeima J, Wachters JE, van der Laan BFAM, Wedman J, Witjes MJH and Halmos GB: Virtual 3D planning of tracheostomy placement and clinical applicability of 3D cannula design: a threestep study. Eur Arch Oto-Rhino-Laryngology 275: 451-457, 2018. PMID: 29177627. DOI: 10.1007/s00405-017-4819-x

3 Szeto C, Kost K, Hanley JA, Roy A and Christou N: A simple method to predict pretracheal tissue thickness to prevent accidental decannulation in the obese. Otolaryngol Head Neck Surg 143: 223-229, 2010. PMID: 20647124. DOI: 10.1016/ j.otohns.2010.03.007

4 Walker RN, Alexander IJ, Sartorius JA and Woomert CA: Anthropometric measurements: Effect of CT depth of pretracheal soft tissue on tracheotomy tube selection. Am J Neuroradiol 33(3): 449-452, 2012. PMID: 22173752. DOI: 10.3174/ajnr.A2823

5 Hess DR: Tracheostomy tubes and related appliances. Respir Care 50(4): 497-510, 2005. PMID: 15807912.

6 Lim PVH and Raman R: Adjustable length tracheostomy tube for the morbidly obese and thick neck patient: A prototype. Otolaryngol - Head Neck Surg 124(1): 56-57, 2001. PMID: 11228454. DOI: $10.1067 / \mathrm{mhn} .2001 .112198$

7 Pandian V, Hutchinson CT, Schiavi AJ, Feller-Kopman DJ, Haut ER, Parsons NA, Lin JS, Gorbatkin C, Angamuthu PG, Miller CR, Mirski MA, Bhatti NI and Yarmus LB: Predicting the need for nonstandard tracheostomy tubes in critically ill patients. J Crit Care 37: 173-178, 2017. PMID: 27756050. DOI: 10.1016/ j.jcrc.2016.05.025

8 Atala A and Yoo JJ: Essentials of 3D-biofabrication and translation. Amsterdam: Elsevier Inc, pp. 43-59, 2015.

9 Eltorai AEM, Nguyen E and Daniels AH: Three-dimensional printing in orthopedic surgery. Orthopedics 38(11): 684-687, 2015. PMID: 26558661. DOI: 10.3928/01477447-20151016-05

10 Qiao F, Li D, Jin Z, Gao Y, Zhou T, He J and Cheng L: Application of 3D printed customized external fixator in fracture reduction. Injury 46: 1150-1155, 2015. PMID: 25702252. DOI: 10.1016/j.injury.2015.01.020

11 Wong KC, Kumta SM, Geel NV and Demol J: One-step reconstruction with a $3 \mathrm{D}$-printed, biomechanically evaluated custom implant after complex pelvic tumor resection. Comput Aided Surg 20(1): 14-23, 2015. PMID: 26290317. DOI: 10.3109/10929088.2015.1076039

12 Zeng C, Xiao J, Wu Z and Huang W: Evaluation of threedimensional printing for internal fixation of unstable pelvic fracture from minimal invasive para-rectus abdominis approach: A preliminary report. Int J Clin Exp Med 8: 13039-13044, 2015. PMID: 26550226.

13 Tahayeri A, Morgan MC, Fugolin AP, Bompolaki D, Athirasala A, Pfeifer CS, Ferracane JL and Bertassoni LE: 3D printed versus conventionally cured provisional crown and bridge dental materials. Dent Mater 34: 192-200, 2018. PMID: 29110921. DOI: $10.1016 / j$.dental.2017.10.003

14 Chung YJ, Park JM, Kim TH, Ahn JS, Cha HS and Lee JH: 3D printing of resin material for denture artificial teeth: Chipping and indirect tensile fracture resistance. Materials (Basel) 11: 113, 2018. PMID: 30248955. DOI: 10.3390/ma11101798 
15 Cheng GZ, Folch E, Brik R, Gangadharan S, Mallur P, Wilson JH, Husta B and Majid A: Three-dimensional modeled T-tube design and insertion in a patient with tracheal dehiscence. Chest 148(4): e106-108, 2015. PMID: 26437816. DOI: 10.1378/ chest. $15-0240$

16 Morrison RJ, Kashlan KN, Flanangan CL, Wright JK, Green GE, Hollister SJ and Weatherwax KJ: Regulatory considerations in the design and manufacturing of implantable 3D-Printed medical devices. Clin Transl Sci 8(5): 594-600, 2015. PMID: 26243449. DOI: $10.1111 /$ cts. 12315

$17 \mathrm{Li} \mathrm{Z}$, Wang C, Qiu W and Liu R: Antimicrobial thiol-eneacrylate photosensitive resins for DLP 3D printing. Photochem Photobiol 95: 1219-1229, 2019. PMID: 30883789. DOI: 10.1111/php.13099

18 Murphy S V and Atala A: 3D bioprinting of tissues and organs. Nat Biotechnol 32(8): 773-785, 2014. PMID: 25093879. DOI: $10.1038 / \mathrm{nbt} .2958$

19 Mäkitie A a, Korpela J, Elomaa L, Reivonen M, Kokkari A, Malin M, Korhonen H, Wang X, Salo J, Sihvo E, Salmi M, Partanen J, Paloheimo KS, Tuomi J, Närhi T and Seppälä J: Novel additive manufactured scaffolds for tissue engineered trachea research. Acta Otolaryngol 133: 412-417, 2013. PMID: 23394221. DOI: $10.3109 / 00016489.2012 .761725$

20 Elsawy MA, Kim KH, Park JW and Deep A: Hydrolytic degradation of polylactic acid (PLA) and its composites. Renew Sustain Energy Rev 79: 1346-1352, 2017. DOI: 10.1016/j. rser.2017.05.143

21 Park JM, Ahn JS, Cha HS and Lee JH: Wear resistance of 3D printing resin material opposing zirconia and metal antagonists. Materials (Basel) 11(6): 1-10, 2018. PMID: 29925763. DOI: 10.3390/ma11061043

22 Lin CH, Lin YM, Lai YL and Lee SY: Mechanical properties, accuracy, and cytotoxicity of UV-polymerized 3D printing resins composed of BisEMA, UDMA, and TEGDMA. J Prosthet Dent 123(2): 349-354, 2020. PMID: 31202550. DOI: 10.1016/j. prosdent.2019.05.002

23 Podgórski M: Structure-property relationship in new photo-cured dimethacrylate-based dental resins. Dent Mater 28(4): 398-409, 2012. PMID: 22136948. DOI: 10.1016/j.dental.2011.11.013
24 Finer Y and Santerre JP: The influence of resin chemistry on a dental composite's biodegradation. J Biomed Mater Res - Part A 69(2): 233-246, 2004. PMID: 15057996. DOI: 10.1002/ jbm.a.30000

25 Rodney J, Ojano-Dirain CP, Antonelli PJ and Silva RC: Effect of repeated tracheostomy tube reprocessing on biofilm formation. Laryngoscope 126(4): 996-999, 2016. PMID: 26267243. DOI: $10.1002 /$ lary.25473

26 Jung H, Kim HS, Lee JH, Lee JJ and Park HS: wound healing promoting activity of tonsil-derived stem cells on 5-fluorouracilinduced oral mucositis model. Tissue Eng Regen Med 17(1): 105-119, 2020. PMID: 32002842. DOI: 10.1007/s13770-01900226-7

27 Gimenez-conti IB and Slaga TJ: The hamster cheek pouch carcinogenesis model. J Cell Biochem Suppl 17F: 83-90, 1993. PMID: 8412211. DOI: 10.1002/jcb.240531012

28Leonhard M, Assadian O, Zumtobel M and Schneider-Stickler B: Microbiological evaluation of different reprocessing methods for cuffed and un-cuffed tracheostomy tubes in home-care and hospital setting. GMS Hyg Infect Control 11: Doc02, 2016. PMID: 26958456. DOI: 10.3205/dgkh000262

29 Perkins J, Mouzakes J, Pereira R and Manning S: bacterial biofilm presence in pediatric tracheotomy tubes. Arch Otolaryngol - Head Neck Surg 130(3): 339-343, 2004. PMID: 15023844. DOI: 10.1001/archotol.130.3.339

30 Teughels W, Van Assche N, Sliepen I and Quirynen M: Effect of material characteristics and/or surface topography on biofilm development. Clin Oral Implants Res 17: 68-81, 2006. PMID: 16968383. DOI: 10.1111/j.1600-0501.2006.01353.x

31 Nandakumar V, Chittaranjan S, Kurian VM and Doble M: Characteristics of bacterial biofilm associated with implant material in clinical practice. Polym J 45(2): 137-152, 2013. DOI: $10.1038 /$ pj.2012.130

Received February 19, 2020

Revised March 27, 2020

Accepted March 28, 2020 